\title{
Needlestick Injuries in Dentistry
}

\author{
Gupta N, ${ }^{1}$ Tak J ${ }^{2}$
}

${ }^{1}$ Department of Periodontics

${ }^{2}$ Department of Oral \& Maxillofacial Pathology

Shree Bankey Bihari Dental College \& Research Centre

Corresponding author:

Neha Gupta

C-401,Sarita Vihar

New Delhi - 110076, India

email: neha.perio@gmail.com

\section{Citation}

Gupta N, Tak J. Needlestick injuries in dentistry. Kathmandu Univ Med J 2011;35(3):208-12.

\begin{abstract}
Needlestick injuries and other sharps-related injuries which expose health care professionals to bloodborne pathogens continue to be an important public health concern. Dentists are at increased risk of exposure to bloodborne pathogens, including Hepatitis B, Hepatitis C, and HIV. This article presents comprehensive information on Needlestick injuries (NSI), post exposure prophylaxis, precautions and suggestions for prevention of NSI in dentistry. Dentists should remember and apply many precautions to prevent the broad spectrum of sharps and splash injuries that could occur during the delivery of dental care.
\end{abstract}

\section{KEY WORDS}

bloodborne, pathogens, needlestick injuries, post exposure prophylaxis

\section{INTRODUCTION}

Serious infections can be transmitted in the dental practice when percutaneous injuries occur. Hepatitis B virus (HBV), hepatitis $C$ virus ( $\mathrm{HCV}$ ), and human immunodeficiency virus (HIV) infections have been recognized as occupational hazards with the risk of transmission from patient to the health care personnel as HCV (3\%), HBV (30\%) and HIV (0.3\%). ${ }^{1-3}$ Needlestick (NSI) injuries is a hidden problem, after it happens, mostly we forget it and get on with our work. Neither the magnitude of the risk of NSI injury nor the practices associated with it have been defined. ${ }^{4-7}$ However, the transmission risk is influenced by the type and number of microorganisms present in the blood, presence of visible blood on the needle, depth of the injury and size and type of needle used. ${ }^{8}$ More than eight million health care workers in the United States work in hospitals and other health care settings and between 0.6 to 0.8 million NSI and other percutaneous injuries occur in them every year, not including the vast number that go unreported. ${ }^{9} \mathrm{NSI}$ are virtually undocumented in developing countries, but probably equal or exceed those in the industrialized world. Inadequate waste disposal system extends the problem beyond the health care settings to cleaners, laundry workers, porters, rag pickers and the general community.

An exposure can be defined as a percutaneous injury (e.g., needle stick or cut with a sharp object) or contact of mucous membrane or no intact skin (e.g., exposed skin that is chapped, abraded, or with dermatitis) that occurs during the course of persons employment, with blood, saliva, tissue, or other body fluids that are potentially infectious. Because accidents with needles are one of the most common types of injury in the health care setting, injuries from needles are often called needle stick injuries.

The 1st Occupational Safety and Health Administration (OSHA) standard specifically written to protect HCV was the 1991 Bloodborne Pathogen Standard. Although tremendous progress has been made as a result, more than 1000 infections caused by 20 pathogens are estimated to be transmitted through sharps or NSI injuries annually. ${ }^{10}$ The greatest threat to dentist are HBV,HCV, HIV infection, and others like syphillis, Blastomycosis, Brucellosis, Cryptococcosis, Diphtheria, Malaria, TB, Prions, Ebola, Herpes, Mumps, Rubella, Rubeola, Infectious Mononucleosis, Influenza, Swine flu and therefore should be evaluation immediately following treatment of the exposure site by a qualified health care professional.

Body fluids are potentially infectious during an occupational exposure

All blood, body fluids, secretions, and excretions except sweat, may contain transmissible infectious agents. Blood contains the greatest proportion of infectious blood borne virus particle titers of all body fluids and is the most critical 
transmission vehicle in the health-care setting. During dental procedures it is predictable that saliva will become contaminated with blood. If blood is not visible, it is still likely that very small quantities of blood are present, but the risk for transmitting HBV, HCV, or HIV is extremely small. Despite this small transmission risk, every dentist should evaluate any occupational exposure to saliva in dental settings, regardless of visible blood.

\section{Risk of infection after an occupational exposure}

\section{Hepatitis B Virus (HBV)}

Dentists who have received hepatitis $B$ vaccine have developed immunity to the virus are at virtually no risk for infection. For an unvaccinated person, the risk from a single needlestick or a cut exposure to HBV-infected blood ranges from $6 \%-30 \%$ and depends on the hepatitis $B$ antigen ( $\mathrm{HBeAg}$ ) status of the source individual. ${ }^{11-14}$ Individuals who are both hepatitis B surface antigen (HBsAg) positive and $\mathrm{HBeAg}$ positive have more virus in their blood and are more likely to transmit HBV. About 95\% of HBV infections will be detectable by the sixth month after exposure. Later seroconversion is rare. Therefore it should be retested at six weeks, three months and six months. There is a significant group of dentists who may not receive an appropriate hepatitis $B$ vaccination, or who have not completed the course, or are non responders to the vaccine, are at significant risk of infection. About $4 \%$ of persons with HBV infection are also infected with Hepatitis Delta virus (HDV).Since HDV is dependent on HBV for replication, immunization to prevent HBV infection, through either pre-or post exposure prophylaxis, can also prevent HDV infection.

\section{Hepatitis C Virus (HCV)}

$\mathrm{HCV}$ is found in saliva but is most frequently acquired by direct blood to blood contact. The estimated risks for infection after a needlestick or cut exposure to HCV infected blood is approximately $1.8 \% .{ }^{15}$ The risk following a blood splash is unknown but is believed to be greater if the source patient is positive for HCV RNA, with no transmission occurring from HCV RNA negative sources. The prevalence of HCV in dental staff has varied from 0 to $6.2 \% .{ }^{15-18}$ No prophylactic measures involving drugs or immunoglobulins are at present available so first aid management is very important. It is essential a baseline sample is taken at the time of injury. No effective vaccination has been developed against $\mathrm{HCV}$.

\section{Human Immunodeficiency Virus (HIV)}

The average risk for HIV infection after a needle stick or cut exposure to HIV infected blood is $0.3 \%$ (about 1 in 300) that is $99.7 \%$ of needle stick exposures to HIV contaminated blood do not lead to infection. ${ }^{16,17}$ The risk after exposure of the eye, nose, or mouth to HIV infected blood is estimated to be, on average, $0.1 \%$ ( 1 in 1,000). The risk after exposure of the skin to HIV infected blood is estimated to be less than $0.1 \%$. A small amount of blood on intact skin probably poses no risk at all. There are no documented cases of HIV transmission due to an exposure involving a small amount of blood on intact skin. The risk is higher if the skin is damaged (for example, by a recent cut), if the contact involves a large area of skin, or if the contact is prolonged. Many of the body fluids like saliva from which HIV has been isolated have not as yet implicated in occupational transmission. The natures of injuries sustained in dentistry are of relatively low risk so risk of HIV infection following sharps injury is low. No effective vaccination is available against HIV.

Types of needles and sharp instruments that can cause injuries are hypodermic needles, IV (intravenous) needles, lancets, razors, scalpels, retractors, suture needles, dental instruments like burs. Low risk injuries occur with needles with narrow lumen that was not placed directly into the vein or artery, no visible contamination of the needle by patient's blood. High risk injuries are deep injury or a penetrating injury by a device visibly contaminated with blood, exposure to a source patient with end stage HIV infection and injury with a needle which had previously been placed directly into source patient's blood vessel. High risk patients who have greater possibility of harboring transmissible viruses include IV drug abusers, patients with multiple sex partners, blood transfusion patients, patients who have lived in Africa or far east and had sex, homosexual patients and patients suffering from jaundice, abnormal weight loss, anorexia and fever. Once the transmission takes place in few cases virus gets eliminated totally by body's immune system whereas others become carrier. In $\mathrm{HBV}$ it is $20 \%, \mathrm{HCV}$ it is $80 \%$ and in HIV it is almost $100 \%$.

Procedures in dentistry are carried out without any anesthesia or under local anesthesia which can make the patient to cause a sudden jerky movement that can lead to increase probability of NSI. Momentary lack of concentration can also result in an injury. Re-sheathing the needle is a common cause of NSI. Incorrectly disposed needle is also a common cause of NSI. Since blood borne pathogens can be transmitted through aerosols generated during certain dental procedures also, NSI has been recently substituted by Inoculation injuries.

\section{Management of Needle stick Injuries}

\section{First aid measures}

\section{Stop all the procedures.}

2. Assess the injury that is the depth of the injury, if the device is contaminated with blood if the needle entered the patient's blood vessel and if the wound bleeding or not. $^{18,19}$

3. Wash with water and soap or a disinfectant for several minutes ( $10 \%$ iodine solution or chloride compounds), do not scrub the affected area since this may inoculate the virus into the tissues, do not suck the affected area, 
encourage bleeding in the area of puncture wound by gentle squeezing above wound to increase venous back pressure. For mucous membrane exposure copious irrigation with tap water, sterile saline or sterile water should be done for several minutes. This reduces the bioburden below the threshold of an infectious dose because of dilution with water.

4. Do not swallow water used for rinsing the mouth.

5. Assess patient's risk factors.

6. Identify the dentist's immune status for HBV.

A. Protected-anti-HBs antibody titre $>100$ IU/L or $10-99$ $\mathrm{IU} / \mathrm{L}$ if last vaccine dose was within 2 years.

B. Incomplete Protection - Anti-HBs antibody titre 10-99 IU/L if last dose $>2$ years. No antibody checks after full primary course or results are unavailable after full primary course. Recommendation- Booster dose of HBV and follow up.

C. Unprotected - No history of immunization with HBV vaccine. Failure to reach greater than $10 \mathrm{IU} / \mathrm{L}$ antibody titre. Incomplete primary course of HBV. RecommendationBooster dose of HBV, give HB immunoglobulin and follow up.

If blood or body substance accidentally gets into the eye it should be irrigated gently and thoroughly with water, without the use of soap. If blood or body substance accidently gets into the mouth then it should be spitted and mouth rinsed several times with water, spitting out after every rinse.

\section{HIV Post Exposure Prophylaxis (PEP)}

It is a short term for anti-retroviral treatment to reduce the likelihood of HIV infection after potential exposure where Post means after, Exposure- a situation where HIV has a chance to get into someone's bloodstream, prophylaxis - a treatment to stop an infection happening. Practical problems and inconvenience involved restrict the dentist from seeking expert advice following NSI. The Centers for Disease Control and Preventions (CDC) PEP guidelines call for treatment with antiviral medications .Time is important and the prophylaxis should start within an hour of exposure. ${ }^{16,20,21}$ Biological rationale of PEP relies on the time interval required for uptake of the virus, processing and incorporation after initial inoculation.PEP cannot prevent the infection but it can prevent the incorporation of the viral DNA into host DNA.Evidence support the use of antiretroviral drugs in PEP. ${ }^{22,23}$ Currently triple combination regimen is employed; usually recommending zidovidine, lamivudine and indinavir for four weeks is used and is believed to be as effective. ${ }^{20}$ Antiretroviral drugs have side effects like nausea, vomiting, anaemia, fatigue, insomnia and renal stone formation. ${ }^{20}$ Also if the virus has already integrated with the host genome then they are ineffective. If the source patient has significant risk factors it is generally appropriate to commence combination ant- retroviral treatment prior to result of HIV test. If the exposure is low but the risk is non negligible, PEP should be given. Initiation of PEP should be the responsibility of an expert in HIV disease and be based upon details provided by the dentist about the type of injury and nature of the source patient. ${ }^{21}$

\section{Prevention of Needle Stick Injuries}

Avoiding occupational exposures to blood is the primary way to prevent transmission of blood borne viruses in dental offices. According to CDC $86 \%$ of NSI can be prevented by using safer devices. Methods used to reduce such exposures in dental settings include engineering and work practice controls and the use of personal protective equipment (PPE), immunization of the staff, education and training for managing the problem in case occupational exposure occurs i.e. the first aid procedures, means of assessing the risk status of the patient. Out of hours access and the contact number of the emergency department should be made available.

Engineering control isolate or remove the blood borne pathogens hazard from the workplace. These are technology-based and incorporate safer designs of instruments and devices. Examples include sharps disposal containers, rubber dams, and self-sheathing anesthetic needles. These controls should be used as the primary method to reduce exposures to blood borne pathogens following skin penetration with sharp instruments or needles.

Work practice controls are behavior-based and are intended to reduce the risk of blood exposure by changing the manner in which a procedure is performed. Examples include using the "scoop" technique to recap an anesthetic needle, removing burs before placing the hand piece in the dental unit, placing sharps containers within reach at eye level in every patients room and and restricting the use of fingers during suturing and when administering anesthesia. Uncapped needle and sharps should not be passed between dentist and the assistant.

Sterilization of hand pieces and burs should be done using disposable pouches and regular checking of the sterilization cycle should be done in the dental offices. Type B autoclave with complete sterilization cycle and drying cycle should be used. Disinfection of the dental chairs between patients, impressions, waterlines and prosthesis should not be ignored.

Standard precautions are the guidelines recommended by the Centers for Disease Control and Prevention for reducing the risk of transmission of blood-borne and other pathogens in hospitals. The standard precautions synthesize the major features of universal precautions (designed to reduce the risk of transmission of bloodborne pathogens) and body substance isolation (designed to reduce the risk of pathogens from moist body substances) and apply them to all patients receiving care in hospitals regardless 
of their diagnosis or presumed infection status. Standard precautions apply to blood; all body fluids, secretions, and excretions except sweat, regardless of whether or not they contain blood; nonintact skin; and mucous membranes. The precautions are designed to reduce the risk of transmission of microorganisms from both recognized and unrecognized sources of infection in hospitals. Worldwide escalation of the use of standard precautions would reduce unnecessary risks associated with health care settings.

Personal protective equipment consists of specialized clothing or equipment worn to protect against hazards. Examples include gloves, masks, protective eyewear with side shields, and gowns to prevent skin and mucous membrane exposures. Double gloves significantly reduce the hand contamination of dentists. ${ }^{24}$ Use of disposable good fitting mask and its change every hour should be followed. Dentist should not work without covering cuts and wounds on hand with water proof band aid.

Education and Training regarding the use of universal precautions when handling body fluids should be conducted. New employees should go through the training program using videos, handouts, posters and interactive sessions, stressing the need to report all injuries, and the availability of chemoprophylaxis. A computerized database of occupational injuries, including percutaneous and NSI involving blood or body fluids should be maintained.

Improving sharps disposal by introducing improved sharps containers in the dental clinics. Sharps should be disposed immediately. Recapping and resheathing should be discouraged. New arrangements for sharps disposal should be done to avoid overfilling boxes.

Sites of starter packs of PEP drugs and/or hepatitis B prophylaxis should be available in the dental offices. Detailed First Aid Procedure following a sharps injury should be displayed. Arrangements for follow-up visits, follow-up testing, records keeping and confidentiality should be made.

\section{Immunization}

Effective vaccination is available against HBV. The course consists of three doses at zero, one month and six months after first dose. ${ }^{2}$ Dentists should be tested for HBs antibodies one to two months after completion of the three dose vaccination series. Those who do not respond or develop the adequate antibody response to the primary vaccine series should go for a second series of the three dose regimen or be tested to determine if they are HBsAgpositive. Most of the non responders do response to the second series of vaccination. WHO recommends "catch up" immunization of high risk groups including health care workers. It should target all the dentists and the dental students. Regular follow up checks for antibody should be done for all the staff of the dental office.
Dentists can be active on several levels in order to help prevent NSI. They can encourage their colleagues, nurses and paramedical staff to address the issue, review NSI data, PEP facilities etc.

\section{CONCLUSION}

Dentists claim compliance with recommended infection control practices and universal precautions against HBV and HIV infection, but most dentists apparently do not adopt universal precautions completely. Adherence to infection control procedures, especially personal protective equipment, has been linked to keeping the incidence of these infections low. ${ }^{25}$ The prevention of NSI is vital because such incidents are one of the most common forms of injury among dentists and represent the most efficient mechanism of transmission of blood borne pathogens. Programmes for the prevention of occupationally acquired infections caused by NSI should include HB vaccination access to HIV PEP and the use of universal precautions and safety devices.

\section{REFERENCES}

1. Klein RS et al. Occupational risk for hepatitis $\mathrm{C}$ virus infection among New York City dentists. Lancet 1991;338:1539-42.

2. Mast EE. Alter MJ. Prevention of hepatitis $B$ virus infection among health care workers. In: Ellis RW, ed. Hepatitis B vaccines in clinical practice. Marcel Dekker,New York.1993:295-307.

3. Elymiyeh B, Whitaker IS, James MJ, Chal Ca, Galea A, Alshafi K. Needlestick injuries in the National Health Service : A culture of silence. JR Soc Med 2004;97:326- 7.

4. Drelich EV. Reducing the risk of needle sticks: methods used to reload syringes. Journal of the American Dental Association 1997;128:55-9.

5. Stewardson DA et al. Occupational exposures occurring among dental assistants in a UK dental school. Primary dental care 2003;10:23-6.

6. Naidoo S. Dentists and cross-infection. Journal of the Dental Association of South Africa 1997;52:165-7.

7. Haiduven DJ et al. A survey of percutaneous/mucocutaneous injury reporting in a public teaching hospital. Journal of hospital infection 1999;41:151-4.

8. Cleveland JL et al. Use of HIV postexposure prophylaxis by dental health care Personnel: an overview and updated recommendations. Journal of the American Dental Association 2002;133:1619-26.

9. National Institute for Occupational Safety and Health (NIOSH) Alert: Preventing needlestick injuries in healthcare settings. Washington DC: NIOSH 1999.

10. Aiken LH,Sloane DM,Klocinski JL.Hospital nurses occupational exposure to blood .Prospective, retrospective, and institutional reports.Am J Public Health 1997;87:103-7.

11. Scheutz F, Mellbye M, Esteban J I et al.Hepatitis B virus infection in Danish dentists;a case control study and follow-up study. Am J Epidemiol 1988;128: 190-196.

12. Porter S R, Scully C. HIV; the surgeons perspective. Br J Oral Max Surg 1994; 32:222-230.

13. Cottone J A, Puttaiah R. Hepatitis B virus infection. Dent Clin North Am 1996; 40:293-307. 
14. Schiff E R, de Medina $M D$, Kline $S \mathrm{~N}$ et al. Veterans administration cooperative study on hepatitis and dentistry. J Am Dent Assoc 1986; 113: 390-396.

15. CDC. Recommendations for follow-up of HCW after occupational exposure to hepatitis C virus. MMWR Morb Mortal Wkly Rpt 1997;46: 603-606.

16. Tokars JI, Marcus R, Culver DH et al.Surveillance of HIV infection and zidovudine use among health care workers after occupational exposure to HIV infected blood:the CDC Cooperative Needlestick Surveillance Group.Ann Intern Med 1993;118: 913-9.

17. ppolito G, Puro V, De Carli G and the ItalianStudy Group on Occupational Risk of HIV infection. The risk of occupational human immunodeficiency virus infection in health care workers. Archs Intern Med 1993; 153:1451-8.

18. Case-control study of HIV seroconversion in health-care workers after percutaneous exposures to HIV-infected blood: France,United Kingdom and United States; Jan1988-Aug 1994. MMWR1995; 44: 929-33.

19. Public Health Laboratory Service AIDS \& STD Centre and Collaborators. Occupational transmission of HIV. Summary of published reports. Dec 1999 ed.
20. Expert Advisory Group on AIDS HIV. Postexposure prophylaxis: guidance from the UK.

21. Chief Medical Officers' Expert Advisory Group on Aids. London: Department of Health, 2000.

22. UK Health Department. Guidance for clinical health care workers: protection against infection with blood borne viruses. Recommendations of the Expert Advisory Group on AIDS and the Advisory Group on Hepatitis. 3rd edition.London: Department of Health 1998.

23. Black R J. Animal studies of prophylaxis. Am J Med 1997; 102: 39-44.

24. Böttiger A, Johansson N G, Samuelsson B et al.Prevention of simian immunodeficiency virus,SIVsm or HIV-2 infection in cynomolgus monkeys by pre and post-exposure administration of BEA-005. AIDS 1997; 11:157-162.

25. Mast S T, Woolwine J D, Gerberding J L.Efficacy of gloves in reducing blood volumes transferred during simulated needlestick injury. J Infect Dis 1993; 168: 1589-1592.USA.

26. Schuman NJ, Owens BM, Turner JE. Survey of hepatitis B exposure and sharps injuries in dental health-care professionals.Compendium of continuing education for dentists 1996,17:986, 990-5. 